PROCEEDINGS OF THE

AMERICAN MATHEMATICAL SOCIETY

Volume 127, Number 5, Pages 1275-1279

S 0002-9939(99)04848-0

Article electronically published on January 27, 1999

\title{
REPRESENTING QUASI-ORDERS BY EMBEDDABILITY ORDERING OF FAMILIES OF TOPOLOGICAL SPACES
}

\author{
A. E. McCluskeY AND T. B. M. McMASTER \\ (Communicated by Alan Dow)
}

\begin{abstract}
An elementary argument constructs, for each cardinal $\alpha$, a topological space whose subspaces, ordered by homeomorphic embeddability, can model every partial order on $\alpha$-many points. We show how to modify this procedure to deal also with quasi-orders (where the antisymmetry condition may fail), obtaining an initial estimate of the cardinality of the space then required.
\end{abstract}

\section{INTRODUCTION}

Of all the simple and elementary notions that lie at the heart of general topology, perhaps the least well understood is ordering by embeddability, the relation introduced into a family of topological spaces by writing $X \hookrightarrow Y$ whenever $X$ is homeomorphic to a subspace of $Y$. This ordering is, of course, reflexive and transitive but it is not antisymmetric in general; thus it is a quasi-order but not (usually) a partial order. To illustrate its subtlety and relative intractability, we refer to the problem of recognising which order-types are those of collections of subspaces of the real line: a complete answer to this natural question seems almost as far away from achievement now as it did three quarters of a century ago, when it was first examined by mathematicians of the stature of Banach, Kuratowski and Sierpiński (see, for example, [1], [2], [8]). Recent years have seen a reawakening of interest in problems of this sort, due in part to their connection with the study of total negation ([3], [4]).

Let us agree to say that a quasi-ordered set $Q$ is realized within a family $\mathcal{F}$ of topological spaces whenever there is an injection $\theta: Q \rightarrow \mathcal{F}$ for which $q \leq q^{\prime}$ if and only if $\theta(q) \hookrightarrow \theta\left(q^{\prime}\right)$. Basic questions are:

(a) which quasi-ordered sets can be realized within the powerset of a given space?

(b) which quasi-ordered sets can be realized within the powerset of a suitably chosen space?

(c) how can we relate the cardinality of the space in (b) to that of the ordered sets?

In connection with (a), it is known that within the powerset of the real line $\mathbb{R}$ one can realize

(i) the cardinal number $\mathbf{c}^{+}[2]$,

Received by the editors August 9, 1997.

1991 Mathematics Subject Classification. Primary 06A06, 54H10.

Key words and phrases. Partial order, quasi-order, ordering by embeddability.

(C)1999 American Mathematical Society 
(ii) the antichain of cardinality $2^{c}[1]$,

(iii) the powerset of $\mathbb{R}$ ordered by set-inclusion [5],

(iv) any quasi-ordered set of cardinality $\mathbf{c}[6]$.

As to (b), it has recently been noted [4] that every quasi-ordered set is realized by subspaces of some space: the argument depending on fairly sophisticated combinatorial and categorical material (see [7]). The main purpose of the present article is to give a direct and self-contained proof of this result, in which the relationship between set and space is sufficiently transparent to allow an initial answer to (c) also to emerge. On the way to this conclusion we deal with the antisymmetric case, where the arguments and conclusions are especially straightforward: every finite poset can be realized by subspaces of a finite space, every poset of infinite cardinality $\alpha$ can be realized within the powerset of a space on $\alpha$-many points.

\section{The PaRtial order CASE}

Lemma 2.1. Suppose given a family $\mathcal{C}$ of $\alpha$-many connected spaces each with $\gamma$ many points, none of which can be embedded into any other. Then there is a space of $\alpha \gamma$-many points whose subspaces realize all $\alpha$-point posets.

Proof. Put $X=\sum_{C \in \mathcal{C}} C$, the topological sum (= disjoint union) of the spaces in $\mathcal{C}$. Given a poset $E$ with $\alpha$-many points, first set up an injection $y \mapsto C_{y}$ from $E$ into $\mathcal{C}$. Now for each $x$ in $E$ define

$$
\theta(x)=\sum_{y \leq x} C_{y}
$$

and it is immediate that $x_{1} \leq x_{2}$ if and only if $\theta\left(x_{1}\right) \hookrightarrow \theta\left(x_{2}\right)$.

Corollary 2.2. Every finite poset is realizable by subspaces of a finite space. Indeed, for each positive integer $n$ there is a space on (at most) $n^{2}$ points whose subspaces realize all n-point posets.

Proof. For each $n \geq 1$ it is easy to exhibit $n$ non-homeomorphic connected topologies on an $n$-element set. This observation is 'sharp' for $n=1$ and $n=2$ but could certainly be improved for larger values of $n$.

Corollary 2.3. There is a countable space whose subspaces realize all countable partial orders.

Proof. By Lemma 2.1, it suffices to find $\aleph_{o}$-many countable connected spaces, none of which is homeomorphic to a subspace of another. One way to do this uses the Stone-Čech compactification $\beta \omega$ of the countably infinite discrete space $\omega$ : we select a sequence $p_{1}, p_{2}, p_{3}, \ldots$ in $\beta \omega \backslash \omega$ so that the subspaces $\omega \cup\left\{p_{1}\right\}, \omega \cup\left\{p_{2}\right\}, \omega \cup\left\{p_{3}\right\}, \ldots$ are pairwise non-embeddable, and then connectify each of these by adjoining a point-at-infinity that lies in the closure of every subset. We shall next explain this in more detail and in more generality.

Lemma 2.4. Let $A$ be a discrete space of infinite cardinality $\alpha$. There is a subset $K$ of $\beta A \backslash A$ such that

(i) $\operatorname{card}(K)=2^{2^{\alpha}}$ and

(ii) whenever $k_{1} \neq k_{2}$ in $K$, then neither $A \cup\left\{k_{1}\right\} \hookrightarrow A \cup\left\{k_{2}\right\}$ nor $A \cup\left\{k_{2}\right\} \hookrightarrow$ $A \cup\left\{k_{1}\right\}$ is true. 
Proof. Fix $p$ in $\beta A \backslash A$. If $A \cup\{p\}$ embeds into $A \cup\{q\}$ (where $q \in \beta A \backslash A$ ), then the embedding must map $p$ to $q$ since the subspaces are discrete everywhere else, and it will be determined by its restriction to the dense set $A$. Since there are $2^{\alpha}$ maps from $A$ to $A$, there are at most that many choices of $q$ that will permit such embedding. For the same reason, each subset of $A \cup\{p\}$ that takes the form $A^{\prime} \cup\{p\}$ where $A^{\prime} \subseteq A$ and $\operatorname{card}\left(A^{\prime}\right)=\alpha$ can be homeomorphic to at most $2^{\alpha}$ many spaces of form $A \cup\{r\}$ where $r \in \beta A \backslash A$; further, there are just $2^{\alpha}$ sets $A^{\prime}$ as described, and so $2^{\alpha}$ is also an upper bound for the number of choices of $r$ that allow $A \cup\{r\} \hookrightarrow A \cup\{p\}$.

For any subset $S$ of $\beta A \backslash A$ whose cardinality is less than $2^{2^{\alpha}}$, it follows that

$\{x \in \beta A \backslash A: \exists s \in S$ such that

$$
\text { either } A \cup\{x\} \hookrightarrow A \cup\{s\} \text { or } A \cup\{s\} \hookrightarrow A \cup\{x\}\}
$$

also has cardinality less than that of $\beta A \backslash A$, and therefore we can choose $y$ in $\beta A \backslash A$ so that $A \cup\{y\}$ is 'incomparable' with all the $A \cup\{s\}$. A routine induction argument now generates a transfinite sequence

$$
\left(k_{\epsilon}, \epsilon<2^{2^{\alpha}}\right)
$$

in $\beta A \backslash A$ whose range $K$ satisfies conditions (i) and (ii).

Note. For each space $X$ let us denote by $C_{o}(X)$ the connected one-point extension $X \cup\{\infty\}$ of $X$ whose topology is merely that of $X$ plus the single extra set $X \cup\{\infty\}$. It is trivial that $X \hookrightarrow Y$ implies $C_{o}(X) \hookrightarrow C_{o}(Y)$, and the converse is also true: if $\theta$ embeds $C_{o}(X)$ into $C_{o}(Y)$, then either $\theta(\infty)=\infty$ or $\theta\left(C_{o}(X)\right) \subseteq Y$, and in both cases the restriction of $\theta$ to $X$ embeds it into $Y$. Lemma 2.4 therefore provides us with the makings of $2^{2^{\alpha}}$ connected pairwise non-embeddable spaces on $\alpha$ points each, and Lemma 2.1 shows how to form, for each cardinal $\gamma$ between $\alpha$ and $2^{2^{\alpha}}$ inclusive, a space on $\gamma$ points whose subspaces realize every partially ordered set on $\gamma$ points. But our interest now lies in extending realizability to quasi-ordered sets of arbitrary cardinality.

\section{THE QUASI-ORDER CASE}

Given an infinite cardinal $\alpha$, choose $\delta=\delta(\alpha)$ to be the smallest cardinal for which there exist $\alpha$-many distinct non-zero cardinals (not necessarily infinite) less than $\delta$, and index these in any fashion as

$$
\left\{\epsilon_{\lambda}: \lambda<\alpha\right\} .
$$

(For example, $\delta\left(\aleph_{o}\right)=\aleph_{o}, \delta\left(\aleph_{1}\right)=\aleph_{\omega_{1}}$.) Then for each cardinal $\epsilon \leq \delta$ we define $D(\epsilon)$ to be the space formed from the topological sum of $\delta$-many copies of the two-element trivial (= indiscrete) space by adjoining $\epsilon$-many isolated points. It is readily seen that $\epsilon<\epsilon^{\prime} \leq \delta$ implies that $D(\epsilon) \hookrightarrow D\left(\epsilon^{\prime}\right)$ and $D\left(\epsilon^{\prime}\right) \hookrightarrow D(\epsilon)$ but that $D(\epsilon)$ and $D\left(\epsilon^{\prime}\right)$ are non-homeomorphic. These simple spaces can now be used as 'cloning agents' to replicate each of the spaces that realize a poset on at most $\alpha$ points, in such a way as to represent any quasi-ordered set of that order of cardinality.

Proposition 3.1. Any quasi-ordered set $Q$ of infinite cardinality $\alpha$ can be realized within the subspaces of a space $X$ whose cardinality does not exceed $\delta(\alpha)$. 
Proof. First, abstract the 'partially ordered skeleton' $P$ of $Q$ by quotienting-out the equivalence relation $\sim$ defined on $Q$ by

$$
q_{1} \sim q_{2} \text { if and only if } q_{1} \leq q_{2} \text { and } q_{2} \leq q_{1},
$$

the set $P$ of equivalence classes being then (unambiguously) ordered by writing

$$
p_{1} \leq p_{2} \text { if and only if } \exists q_{1} \in p_{1}, q_{2} \in p_{2} \text { such that } q_{1} \leq q_{2} .
$$

Since $P$ is then a poset on at most $\alpha$ elements, our previous discussion shows how to realize it via an association

$$
p \mapsto \theta(p)=\sum_{y \leq p} C_{y}
$$

where each $C_{y}$ is of the form $C_{o}(A \cup\{r(y)\})$ and $r(y) \in \beta A \backslash A$. We also index the elements of $Q$ that belong to each element $p$ of $P$ :

$$
p=\left\{p_{\lambda}: \lambda<\zeta(p)\right\}
$$

where $\operatorname{card}(p)=\zeta(p) \leq \alpha$. Now let $X$ be the topological sum

$$
\sum_{y \in P}\left(C_{y} \times D(\delta)\right)
$$

which is a space of cardinality $\delta(\alpha)$, and represent $p_{\lambda}$ by its subspace

$$
\phi\left(p_{\lambda}\right)=\theta(p) \times D\left(\epsilon_{\lambda}\right)=\sum_{y \leq p}\left(C_{y} \times D\left(\epsilon_{\lambda}\right)\right) .
$$

Since each element of $Q$ is uniquely expressible in the form $p_{\lambda}, \phi$ is a mapping from $Q$ into the powerset of $X$; we claim that it is one-to-one, and that

$$
p_{\lambda} \leq p_{\lambda^{\prime}}^{\prime} \Leftrightarrow \phi\left(p_{\lambda}\right) \hookrightarrow \phi\left(p_{\lambda^{\prime}}^{\prime}\right) .
$$

(1) If $p_{\lambda} \leq p_{\lambda^{\prime}}^{\prime}$ then $p \leq p^{\prime}$ which implies that $\theta(p) \subseteq \theta\left(p^{\prime}\right)$. So both $\theta(p) \hookrightarrow \theta\left(p^{\prime}\right)$ and $D\left(\epsilon_{\lambda}\right) \hookrightarrow D\left(\epsilon_{\lambda^{\prime}}\right)$ are satisfied, and it is immediate that $\phi\left(p_{\lambda}\right) \hookrightarrow \phi\left(p_{\lambda^{\prime}}^{\prime}\right)$.

(2) We begin the converse with the elementary observation that if $T$ is a trivial space, $U$ is any space and $A$ is a subset of the product $U \times T$ such that no two points of $A$ have the same first coordinate, then projection parallel to $T$ homeomorphically embeds $A$ into $U$. Suppose now that there is an embedding $e$ of $\phi\left(p_{\lambda}\right)$ into $\phi\left(p_{\lambda^{\prime}}^{\prime}\right)$. The image under $e$ of the connected subset $K=C_{p} \times\{s\}$ of $\phi\left(p_{\lambda}\right), s$ being one of the isolated points of $D\left(\epsilon_{\lambda}\right)$, must lie within one of the components of $\phi\left(p_{\lambda^{\prime}}^{\prime}\right)$. This component is of one of the two forms $C_{y} \times\{t\}$ or $C_{y} \times T$, where $y \leq p^{\prime}$ and $t$ is an isolated point in $D\left(\epsilon_{\lambda^{\prime}}\right)$ and $T$ is a two-point trivial space. Since $K$ is $T_{o}$, its copy $e(K)$ could not have two points in $C_{y} \times T$ with the same first coordinate, so in both cases we deduce that $C_{p}$ embeds into $C_{y}$, that is, $C_{o}(A \cup\{r(p)\}) \hookrightarrow C_{o}(A \cup\{r(y)\})$. As noted above, this forces $A \cup\{r(p)\} \hookrightarrow A \cup\{r(y)\}$ which in turn implies $r(p)=r(y)$ and $p=y$, yielding $p \leq p^{\prime}$. So indeed $p_{\lambda} \leq p_{\lambda^{\prime}}^{\prime}$ follows.

(3) If $\phi\left(p_{\lambda}\right)=\phi\left(p_{\lambda^{\prime}}^{\prime}\right)$ then (2) shows first that $p=p^{\prime}$. But then the numbers of components in $\phi\left(p_{\lambda}\right)$ and $\phi\left(p_{\lambda^{\prime}}^{\prime}\right)$ that are homeomorphic to $C_{p}$ will have to be equal, and these numbers are $\epsilon_{\lambda}$ and $\epsilon_{\lambda^{\prime}}$. Thus $\lambda=\lambda^{\prime}$, and $p_{\lambda}$ is indistinguishable from $p_{\lambda^{\prime}}^{\prime}$.

This concludes the demonstration.

Corollary 3.2. There is a countable space whose subspaces permit the realization of every countable quasi-ordered set. (Compare Corollary 2.3.)

Proof. $\delta\left(\aleph_{o}\right)=\aleph_{o}$. 
Comments. It would be good to know:

(i) how much the $n^{2}$ estimate in Corollary 2.2 could be reduced,

(ii) if more careful connectification would be enough to produce spaces with better separation than $T_{o}$ for the realization of posets,

(iii) where to find cloning agents with some separation and with lower cardinality than $\delta(\alpha)$.

\section{ACKNOWLEDGEMENTS}

It is a pleasure to record our indebtedness to Stephen Watson (York, Ontario), Tony Wickstead (Queen's, Belfast) and the (anonymous) referee of TOPOSYM 1996 for valuable conversations and insights that have contributed to this article.

\section{REFERENCES}

[1] C. Kuratowski, Sur la puissance de l'ensemble des "nombres de dimension" au sens de M. Fréchet, Fund. Math. 8 (1926), 201-208.

[2] C. Kuratowski and W. Sierpiński, Sur un problème de M. Fréchet concernant les dimensions des ensembles linéaires, Fund. Math. 8 (1926), 193-200.

[3] P.T. Matthews and T.B.M. McMaster, Families of spaces having prescribed embeddability order-type, Rend. Ist. Mat. Univ. Trieste 25 (1993), 345-352. MR 96e:54015

[4] P.T. Matthews and T.B.M. McMaster, Quasiorder contexts for total negation, Proc. Eighth Prague Topological Symp. (1996), 283-287. CMP 98:13

[5] A.E. McCluskey, T.B.M. McMaster and W.S. Watson, Representing set-inclusion by embeddability among subspaces of the real line, to appear in Top. Appl.

[6] A.E. McCluskey and T.B.M. McMaster, Realizing quasiordered sets by subspaces of 'continuum-like' spaces, submitted to Order.

[7] A. Pultr and V. Trnková, Combinatorial, Algebraic and Topological Representations of Groups, Semigroups and Categories, North-Holland, Amsterdam (1980). MR 81d:18001

[8] W. Sierpiński, Sur un problème concernant les types de dimensions, Fund. Math. 19 (1932), 65-71.

Department of Mathematics, National University of Ireland, Galway, Ireland

E-mail address: a.mccluskey@ucg.ie

Department of Pure Mathematics, Queen's University of Belfast, Belfast BT7 1NN, NORTHERN IRELAND

E-mail address: t.b.m.mcmaster@qub.ac.uk 